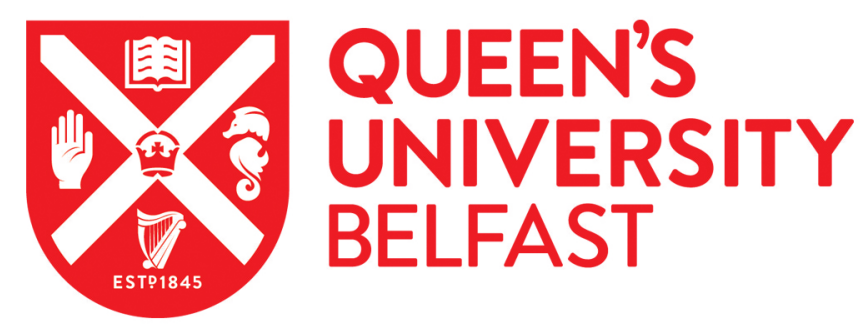

\title{
MyEvents: A Personal Visual Analytics Approach for Mining Key Events and Knowledge Discovery in Support of Personal
} Reminiscence

Parvinzamir, F., Zhao, Y., Deng, Z., \& Dong, F. (2019). MyEvents: A Personal Visual Analytics Approach for Mining Key Events and Knowledge Discovery in Support of Personal Reminiscence. Computer Graphics Forum, 38(1), 647. https://doi.org/10.1111/cgf.13596

Published in:

Computer Graphics Forum

Document Version:

Peer reviewed version

Queen's University Belfast - Research Portal:

Link to publication record in Queen's University Belfast Research Portal

\section{Publisher rights}

(c) 2019 The Authors Computer Graphics Forum (c) 2019 The Eurographics Association and John Wiley \& Sons Ltd. This work is made available online in accordance with the publisher's policies. Please refer to any applicable terms of use of the publisher.

\section{General rights}

Copyright for the publications made accessible via the Queen's University Belfast Research Portal is retained by the author(s) and / or other copyright owners and it is a condition of accessing these publications that users recognise and abide by the legal requirements associated with these rights.

Take down policy

The Research Portal is Queen's institutional repository that provides access to Queen's research output. Every effort has been made to ensure that content in the Research Portal does not infringe any person's rights, or applicable UK laws. If you discover content in the Research Portal that you believe breaches copyright or violates any law, please contact openaccess@qub.ac.uk. 


\title{
MyEvents: A Personal Visual Analytics Approach for Mining Key Events and Knowledge Discovery in Support of Personal Reminiscence
}

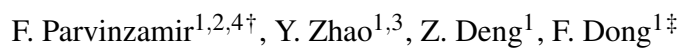 \\ ${ }^{1}$ University of Bedfordshire, Centre for Visualisation and Data Analytics, Luton, United Kingdom \\ 2 Queen's University Belfast, School of Electronics, Electrical Engineering and Computer Science, Belfast, United Kingdom \\ ${ }^{3}$ Communication University of Zhejiang, Hangzhou, China \\ ${ }^{4}$ InsightZen Group, Hangzhou, China
}

\begin{abstract}
Reminiscence is an important aspect in our life. It preserves precious memories, allows us to form our own identities and encourages us to accept the past. Our work takes advantage of modern sensor technologies to support reminiscence, enabling self-monitoring of personal activities and individual movement in space and time on a daily basis. This paper presents MyEvents, a web-based personal visual analytics platform designed for non-computing experts, that allows for the collection of long-term location and movement data and the generation of event mementos. Our research is focused on two prominent goals in event reminiscence: 1) selection subjectivity and human involvement in the process of self knowledge discovery and memento creation; and 2) the enhancement of event familiarity by presenting target events and their related information for optimal memory recall and reminiscence. A novel multi-significance event ranking model is proposed to determine significant events in the personal history according to user preferences for event category, frequency and regularity. The evaluation results show that MyEvents effectively fulfils the reminiscence goals and tasks.
\end{abstract}

\author{
CCS Concepts \\ -Computing methodologies $\rightarrow$ Significant ranking; $\bullet$ Visual Analytics $\rightarrow$ Data mining, Visualisation; $\bullet$ Design $\rightarrow$ Visual com- \\ ponent design;
}

\section{Introduction}

Reminiscence is the act of recalling memory of past events and experiences. It constitutes a very important part in our life in terms of preserving precious memories, forming our own identities and accepting the past [Lew71]. Studies on episodic memory suggest that a constant review of personal life helps improve emotions with families and friends [BSK05], and allows us to seek solutions for present issues by looking into past experiences [WM99]. Reminiscence is also highly valuable for medical treatment in mental health [HW03], offering therapies to conditions such as memory impairment and depression.

Mementos are objects that are kept as a reminder of significant experience in the past. Prior work on physical mementos shows the importance of everyday objects for reminiscence [PWB08]. Digital mementos have attracted significant attention in recent years

\footnotetext{
$\dagger$ F.Parvinzamir@qub.ac.uk

₹ Feng.Dong@beds.ac.uk
}

[KW11,NGN08,PBW14,PW10,vdH14,WKP*12]. Compared with many of the previous works, this paper casts its focus on the recall of a set of key events that an individual experienced in their personal history, thus facilitating a significant part of reminiscence by bringing back memories at key locations such as home, school, work place, holidays and other activity venues. Event mementos also provide important contextual information for object mementos, such as a souvenir bought during a holiday trip or a gift from a friend during dinner in a restaurant. Our work takes advantage of modern sensor technologies (e.g. smartphones with built-in GPS sensor, etc.), which nowadays enable self-monitoring of personal activities on a daily basis, leading to a spectrum of personal location and movement data along a personal lifespan to support longterm memory. However, given the huge amount of data captured over a lengthy duration, it is difficult for the individuals to find out desired information from their own data. Studies of digital archives also show that collections of large and poorly organised digital objects often become invisible and inaccessible over the course of time [WBC10]. To this end, visual analytics can be of great as- 
sistance in terms of supporting effectual organisation, search and utilisation of such data for reminiscence. Compared with the previous work on similar topics [BSSB10, TBHC16], MyEvents offers an environment that allows for the analysis of a long-term location and movement data, from which event-mementos can be generated through personal visual analytics. Our research is focused on the improvement of the following aspects in event reminiscences: 1) event selection subjectivity and human involvement in the process of self-knowledge discovery and memento creation, by using a novel data mining model; and 2) the enhancement of event familiarity by visualising target events and their related information for optimal memory recall and effective reminiscence.

Human participation plays a key role in reminiscence. Researchers have suggested that autobiographical memory is not a place that simply stores all previous events, rather, it is a subjective interpretation of the past. People like to be actively involved in the process of selecting and organising mementos [FKP*02] and providing narrations to support reflection and emotion. In particular, studies have demonstrated that personal contextual information is much more relevant in reflecting on memory than impersonal news [RCDH03].

MyEvents is hence designed to support the active involvement of individual users in reminiscence by searching and selecting from personal location and movement data, acquired using mobile technologies over a long period of time, to generate event mementos. Personal visual analytics is designed for interactive self knowledge discovery, as well as exploration of targeted events and their information from the lengthy data. This also belongs to the area of "casual information visualisation" [PSM07] in which the targeted end users are normal citizens instead of computing experts.

As a platform designed to support reminiscence, MyEvents offers an integrated environment of data analytics, visualisation and human-computer interaction, featuring new techniques to support selection subjectivity from daily events, and novel visual presentations for event mementos to evoke event familiarity. To summarise, the main technical contributions of MyEvents include:

- A novel multi-significance event ranking model. This identifies significant events in personal history according to user preferences through ranking. This allows users to efficiently identify key events over a selected period of time based on their personal preference settings to create mementos, including the preference for event category, occurrence frequency and regularity. The frequency preference enables users to make choices between frequently or infrequently visited places. The regularity preference models the occurrence changes. Through the event category preference, the users may set priorities for the types of events in their query.

- An interactive visualisation to support heuristic search and discovery. This includes the following: timeline and map-based visualisations to allow the users to gain an overview of personal events at the selected time period; a search bar that allows the users to seek events with multi-keywords including name, category and preferences via multiple and heuristic keyword entry; hints based on each of the newly entered keywords to guide the search; and a control panel that controls the event search via a graphical interface.
- A novel visual representation of event mementos. This visually encodes a set of heterogeneous information about the event, including time, photos, location, statistics and contextual information. Associations with other events (e.g. events on the same day) are highlighted to further enhance memory reflection.

Altogether, MyEvents supports interactive queries and mining for significant events, to support the process of reminiscence. The user interface consists of: a search box, MyMoment, which is an interactive presentation of event mementos, and a control panel, Eventline, which gives an overview of all events with an event category legend (Figure 1). However, investigating any medical terms such as improvement in memory impairment or depression is beyond the scope of this paper.

\section{Related Work}

In MyEvents, personal location data are essentially time-varying data with semantic labels. In this section, we review the most related research areas to our work which are reminiscence visualisation, personal visual analytics, time-oriented event visualisation and spatiotemporal visual analytics.

\subsection{Reminiscence visualisation}

The work in reminiscence visualisation is limited. LastHistory [BSSB10] focuses on temporal patterns in personal music listening histories to facilitate reminiscing. An interactive timeline matrix is used to visualise everyday music listening history on a 24 hour basis together with contextual personal information such as photos and calendar events. Daily-streamed songs are lined up vertically on a timeline in colour-coded circles to represent the genres and songs ranked based on frequency of listening. The interaction shows the links to similar songs and song sequences to help reminiscing and mood analysis. The frequency-based song-ranking algorithm is helpful for mood analysis. Dias [DFG12] uses a different timeline-based layout for the visualisation of music-listening history using stacked dots. Filtering is provided to view only the selected songs and the ranking is purely frequency-based. AppInsight [BNG12] presents a visualisation tool that helps reminiscing about computer software usage history with an hourly timeline matrix. A duration-based usage ratio is used to rank the software apps. Details of app usage can be obtained and comparisons of several apps are supported from the visual interface. None of the above work is designed to reveal important events from a large number of trivial events in daily life-logging data. In contrast, MyEvents strives to address the problem by proposing a novel importancebased ranking algorithm, mRank.

Our work is close to VisualMementos [TBHC16], which integrates a timeline with a map followed by semantic clustering of GPS logs to analyse and visualise personal movements at different temporal and spatial scales, hence supporting reminiscing for selfreflection and memento sharing. They use different sizes of circular map segments along a time axis in chronological order to illustrate visits or repeated visits and associated duration within a geographical area. In comparison, MyEvents casts more focus on selection subjectivity: the mRank multi-significance event ranking together 
with the interactive visualisation are designed to support users' involvement in the creation process of the mementos by facilitating their selection of key events in their personal history for reminiscence. Meanwhile, MyEvents involves additional contextual information in the visualisation of the event mementos to evoke event familiarity, such as other events which occurred on the same day. Such contextual information is proved to be important in terms of helping memory recall. Finally, the data mining and visualisation in MyEvents offer more semantically meaningful outcomes through the provision of event category information.

\subsection{Personal visualisation and visual analytics}

Reminiscence visualisation is strongly related to personal visualisation and visual analytics. Unlike the analysis of population data, which is very common in applications such as trajectory mining and visual analytics, personal visual analytics focuses on the visualisation and analysis of personal data. Huang et al. [HTA*15] present an extensive survey of personal visualisation approaches that support data analysis in a personal context from a wide range of sources, such as music streaming histories [BSSB10], movements [KW11, TBHC16], computer usage [BNG12], email contents and archives [VGD06, HK09]; the review covers a wide variety of aspects in daily life, such as ecological footprint, energy consumption, nutrition, social life and health. Vrotsou uses data mining to find patterns in diaries for a group of people [VEC09, VJC09]. Though the work is not specially designed for personal visual analytics, it is closely related to daily life logging and analysis. Compared to these works, MyEvents is designed for the visual analytics of location and movement data with a focus on facilitating the selection and presentation of key personal events for reminiscence. The mRank model in MyEvents allows enhanced engagement by users by encoding the selection of user preference in the model for event ranking and hence supporting meaningful knowledge discovery.

\subsection{Time-oriented event sequence visual analytics}

Our work is also related to the visual analytics of time-oriented data, which has been an important research field in visualisation for several decades [AMM*07]. Most of the existing methods have been used in applications such as personal health, streaming songs, computer-logging and population. Rind et al. [RWA*13] and West et. al [WBH15] have presented a detailed review of visual analytics techniques for electronic health records (EHRs), which is one of the most studied time-oriented data types in the visualisation community. Lifelines [PMR ${ }^{*}$ ]6] is a pioneer work in the visualisation of individual patient records, which provides a general visualisation environment for clinical problems, diagnoses, test results or medications using timelines. Lifelines2 [WPS*09] describes the visualisation of temporal categorical data across multiple records to assist doctors to view and discover patterns across these clinical records for hypothesis generation, and to find causeand-effect relationships in population data. LifeFlow [WGGP*11] and EventFlow [MLL*13] are tools for event sequence analytics from a group of patients. They extract and highlight common event sequences from patient records. VISITORS [KSTM10], which is based on KNAVE [SC99] and KNAVE II [SGBBT06], uses aggregation to extract meaningful interpretations from multiple patients' raw time-oriented data. PatternFinder [FKSS06] provides tools for the user to query patterns by specifying the attributes of events and time spans. Outflow [WG12] and DecisionFlow [GS14] use Sankey-based visualisation [RHF05] to help visualise and analyse the causal relationships of events in complex event sequences. Clustering and inverse document frequency are also used to extract significant events in their work.

\subsection{Spatiotemporal visual analytics}

Most of the work in the spatiotemporal visual analytics field is on visual analysis of massive trajectory data. Andrienko et al. [AAB $\left.{ }^{*} 13\right]$ provide a detailed description of work on spatiotemporal data visual analytics. Krueger et al. [KTE14] introduce context data into trajectory data analysis; their system identifies potential places from a large group of raw trajectory data and adds contextual information such as name and category. Yu et al. [YWL*15] are able to distinguish home and work places from public transportation data. Von Landesberger et al. [vLBR*16] combine spatial and temporal simplifications for graph-based visual analysis. Beecham et al. [BWB14] study commuting behaviour by visually analysing the London Cycle Hire Scheme data. [CYW*16] discover movement patterns by analysing geo-tagged web text data of a large population. Andrienko et al. [AAH*13] extract significant places from population trajectory data. Recently, they began to study privacyrespectful discovery of place semantics [AAFJ16]. As trajectory data often do not come with semantic information, most of the existing works focus on pattern mining from the trajectories of a large population or the automatic generation of semantic information.

The main research focus of MyEvents in time-oriented and spatiotemporal visualisation is to offer services for informed user query and exploration of key events in personal history for reminiscence. The interactive visualisation is based on a multiscale timeline, enabling users to explore data from different time periods, and allowing them to gain overviews of relevant events based on their queries; events with high significance ranking are highlighted, facilitating the subjective selections.

\section{Description of MyEvents}

\subsection{Definition and data}

Within the scope of this work, we define an event as an activity that takes place at a physical location on a particular date and time, for example, shopping at a local shopping centre on 7th July 2015, or studying at a local library on 12th April, 2016. An event is repeated by revisiting the same place, e.g. 10 revisits to the local library. Notably, here we interchange the concept of event and activity by assuming that the same activity always happens at the same place, for example studying in the library, and eating in the restaurant. Such an assumption is reasonable to a certain extent and it is made due to the lack of information about the activity within one place. This can be improved in future work when more activity data are made available. Trajectory data can be collected by various sensors. In this case, such data is obtained by means of smartphones with a GPS sensor and an individual tracking application called 
Moves [Oy12] This automatically acquires personal location and movement data on a daily basis. The data from the mobile applications such as Moves have already undergone initial preprocessing by removing outliers and detecting movements and stops. The data include location information such as place names, coordinates, duration, time, and movement information such as temporal tracking points, and movement types (walking, running, cycling and transport). The Moves data can be accessed via APIs or downloaded as files in standard data formats such as JSON. However, the place names and the movement types provided by Moves constitute lowlevel semantic information in the data. The user can manually annotate the data with high level semantic information such as actual name and category via the provided Foursquare venue APIs within the app. In our case, the data has been almost entirely annotated by users - see Listing 1 .

The event ranking model presented in this paper (see Section 4) is based on the annotation of the data, which are made available either automatically via APIs or through manual annotation by the users. The annotation gives the name of an event within a specific location; for example, "exam" takes place at "university". The ranking model works out the occurrence of these events and computes their importance according their frequency, regularity and categorisation - more details can be found in Section 4.

Each event $e$ embodies the following attributes in Table 1. These attributes can be used for the significant event ranking and validation.

\begin{tabular}{lll}
\hline Attribute & Symbol & Data Type \\
\hline start_time & $e_{t s}$ & datetimeoffset \\
end_time & $e_{t e}$ & datetimeoffset \\
name & $e_{n}$ & String \\
\hline Local_id & $e_{i d}$ & local UUID \\
Category & $e_{\text {Cat }}$ & Event Category $<$ String $>$ \\
\hline Location & $e_{\text {LatLng }}$ & Coordinate<Float $>$ \\
Foursquare_id & $e_{f i d}$ & place UUID \\
Foursquare_category & $e_{c a t i d}$ & Foursquare place Cat UUID \\
\hline
\end{tabular}

Table 1: The data attributes and types

\subsection{Design goals and requirements}

Our design goal is to support personal memory recall for reminiscence by creating event mementos and interactive visual analytics for personal data, with particular focus on two goals: event selection subjectivity and familiarity. The former is related to personal involvement and user experience in the reminiscence process in terms of selecting targeted events for event mementos, while the latter refers to the presentation of the targeted events to evoke memory familiarity.

Research on autobiographical memory has highlighted the importance of subjectivity in reminiscence, as human memory is not an objective storage of all previous events; instead, it is a personal self-reflection that requires significant involvement from human subjects to decide the events that reflect their best memories. While the locations and movement data are available through selfmonitoring sensors, users need support in order to retrieve and revisit their personal history from the big data and make decisions

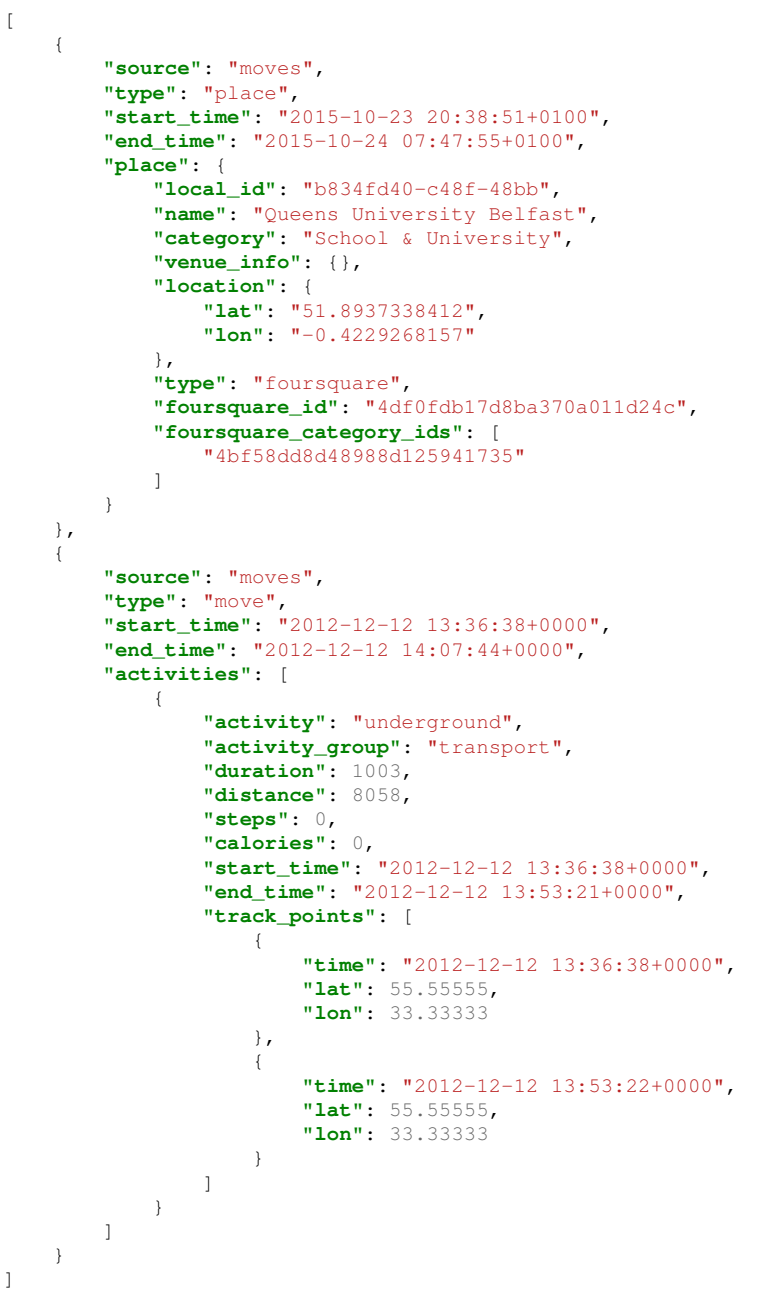

Listing 1: The structures of the preprocessed data from Moves

about their memorable places. MyEvents is designed for this purpose.

As MyEvents is designed for personal reminiscence, the targeted end-users are average citizens who are not familiar with information visualisation. To reflect this, we employ strategies to ensure the design of a simple and intuitive user interface. In fact, the interactive review of personal history itself is also part of a reminiscence process, and one of the missions of MyEvents is to offer a sound interactive tool to make the users enjoy this personal memory recall process through self-knowledge discovery and visualisation. Our design aims to strike a balance between the level of automation and user control, allowing users to interactively seek desired information based on their own preferences, while being supported by data mining techniques to achieve efficient self knowledge discovery and information retrieval.

To fulfil the above goals, we have identified the following key user requirements through methods such as questionnaires and interviews in line with user centred design and agile processes [KSDK11, SMM12], and through literature reviews as described 


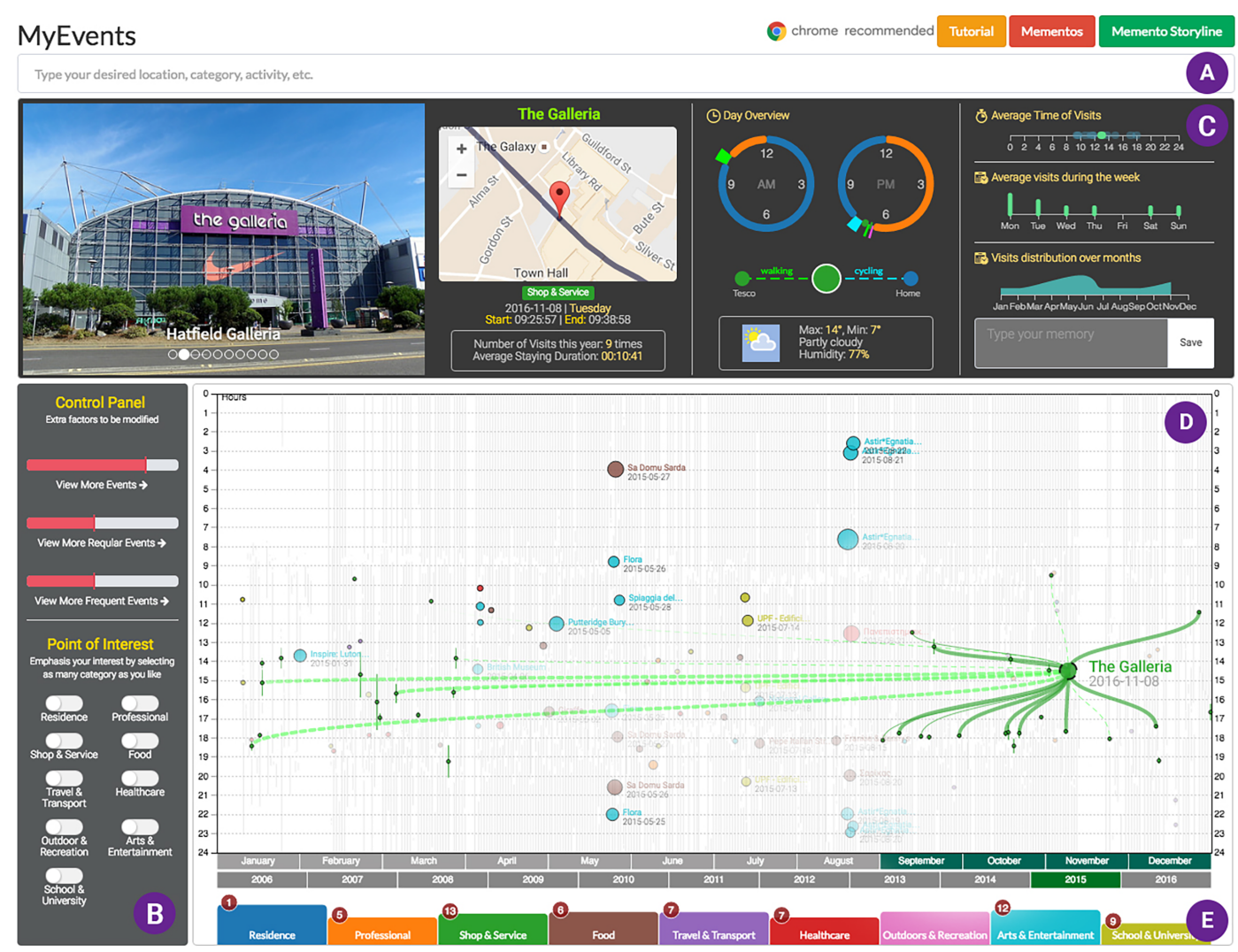

Figure 1: The main interface of MyEvents: A) Search box for event query; B) MyMoment: an interactive presentation of event mementos; C) control panel; D) Eventline: visualisation of events along a timeline with indications of their significance ranking; E) event category legend

in Section 2. The iterative evaluations targeted user-centred design goals, in which users' opinions and preferences are embedded in the process of design and implementation, in order to determine the best way to represent the extracted knowledge and information. Each questionnaire contained different tasks to test usability, user interface satisfaction and system reliability, in order to determine the accuracy, perceptibility, design, and intuitiveness of our approach. Moreover, the interviews included a set of general questions to understand the participants' opinions about the approach, the level of knowledge they had gained during the process, and any desired features that might help coherent memory recall.

\subsubsection{Event selection subjectivity and human involvement (G1)}

This goal addresses active human participation within personal reminiscence, since the process of recalling autobiographical memory is a highly subjective interpretation of the past. Hence, MyEvents should support this active involvement of individual users to create event mementos.

Gain overviews (R1): Users need to have an overview of all the personal events within a selected period of time. The overview should be available before a user drills down for more details in the data. Hence, MyEvents needs to follow the overview-first, detailson-demand visualisation methodology introduced by [Shn96].
Enquiry-based retrieval (R2): In a fashion similar to many online search services (e.g. Google), users are often keen to use natural languages that may involve a number of keywords to customise enquiries about their personal history, such as names and categories of event location, date and time etc. They are also interested in discovering events that occurred frequently or infrequently, regularly or irregularly. For example, a frequently visited place could be their previous home, school or workplace, and an infrequently visited place could be associated with a holiday trip. Also, users need to be able to adaptively adjust the search results using the clues provided by the system.

Control panels (R3): This should also be made available to users who prefer to set their search targets and preferences via graphical user interface components. Users should be able to define points of interest together with their level of significance within the control panel.

Events visualisation and highlights (R4): Location and time information should be made available in the event visualisation. Important events need to be highlighted to facilitate user selections. Users need to define the importance of an event according to its name, category, date and time together with preference settings, e.g. frequent or infrequent, regular or irregular. The system needs to highlight highly ranked events according to the calculations based on user input. Events with low rankings should be faded-out in the 
visualisation to avoid over-plotting. Users may also wish to view the associations between the events, for example, repeated and sequential events.

Data upload (R5): We need to allow users to upload their personal data. The platform needs to be equipped with a database that enables the storage of long-term personal data as well as the created mementos from multiple users.

Create, save, and retrieve mementos (R6): The platform needs to allow users to save their digital mementos for re-access in the future. They should be able to download created mementos from the system to share with their friends.

\subsubsection{Event familiarity (G2)}

This goal considers the presentation of events and their notable information interactively to facilitate self knowledge discovery and exploration of targeted events. Event familiarity helps the recall of memory and the creation of mementos that may be used in support of reminiscence.

Contextual details (R7): We need to allow users to view event information according to their location, date and time. In addition, contextual information about the events, including indications of other events that occurred on the same day, are also desirable to users.

Photos (R8): Photos taken by users during the events or photos from the public to show the event venue are very useful in terms of supporting memory recall.

Statistics (R9): Statistical information about the event is helpful by helping users recall how often similar events occurred. This may include average duration, average duration during the week, and distribution of the occurrence over months.

\subsection{System overview}

MyEvents is designed according to the goals and requirements discussed in the previous section. The system is composed of four modules, namely data repository, data analysis, visualisation, and interaction. The data repository accommodates the personal lifetracking data and the saved mementos. The security of the data repository is built into the core of the system to protect users' sensitive data. The server is located in a secured cloud service in the $\mathrm{UK}$, which is in compliance with the regional legislation and data protection laws. The HTTPS protocol is enforced for the whole platform to protect users' privacy. Authentication and authorisation is built on the Spring Security framework, which is the most widely used Java web security solution and tested by millions of users around the world.

The data analysis module includes the preprocessing of the raw data and the event ranking computation. The visualisation represents the output from the data analysis module. It includes the event visualisation (EventLine), geographical map, MyMoment, memento storyline and memento list. The user interaction has a direct impact on the visualisation and the event ranking. It can allow users to explore events, get additional information, customise the event ranking (by using the control panel and search box), and save or retrieve the mementos - see Figure 2.

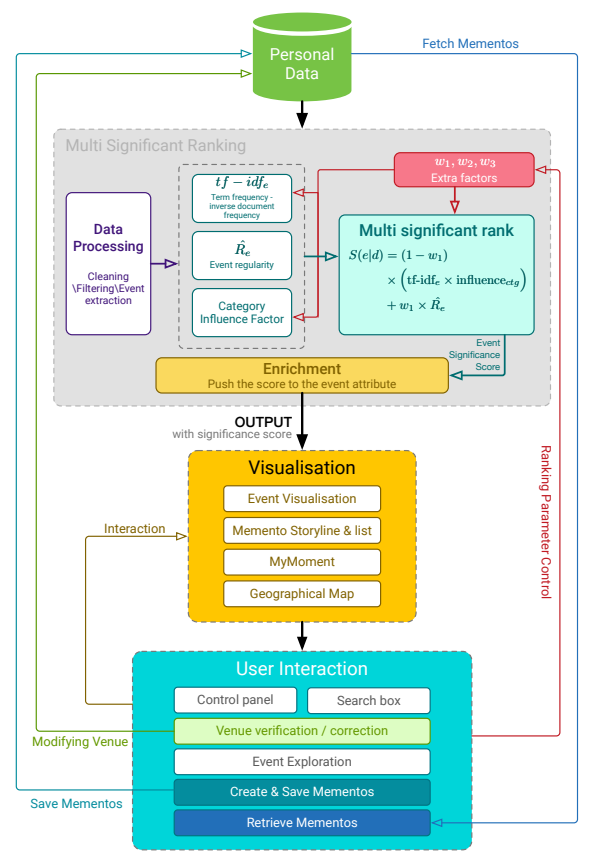

Figure 2: MyEvents system overview

\subsection{User interface and interaction}

The user interface of MyEvents is composed of a search bar, a control panel and a number of components for event visualisation. While the search bar and control panel are designed for usercontrolled event retrieval, the components for event visualisation constitute the key to the event mementos. The event memento is designed to present details of an event in terms of its time, location and duration etc. in order to support best memory recall. The specific components of the event visualisation include the EventLine, map, MyMoment and event category legend.

\subsubsection{User interface}

Search box: The search box is designed to allow event queries using keywords for named entities in natural language. This meets the requirements from non-computing expert users who are keen to make queries about events according to their place names, time, category and other preference settings such as frequency and regularity - see (R2).

The search box offers smart assistance for the search. The search can be incremental, allowing for entry of multiple named entities. Auto-suggestion is provided to assist the search - see Figure (1 A). The named entities (i.e. place names, categories, time and day) plus the search preference can be entered in an arbitrary order to facilitate the user search.

Control panel: The control panel is also designed as an alternative means to search, in which the users can set the search parameters via a graphical user interface - see (R3). More specifically, as shown in Figure $(1-\mathrm{C})$, three sliders are made available to allow users to control how many events to visualise, view more or less 
regularly occurred events, and see more or less frequently occurring events. This adjustment is supported by the underlying mRank model that gives ranking to the events, which subsequently decides the size of the event icons in the event visualisation.

Map: The map shows the geographical locations of the significant events extracted by the mRank by using simple markers. This provides a simple overview of the location of all the significant events - see (R4).

EventLine: The EventLine is a major visualisation component to present personal events along a multiscale timeline. A multiscale timeline shows time/data information in years or months through two layers. The user can select a year to display the events in 12 months. The month is not selectable but is highlighted to show which months the selected event occurred.

The Eventline offers an overview of all the events in a selected period by displaying them along the timeline in light grey colour see R1. It visualises the events according to their significance calculated using the mRank model - see (R4). More specifically, the events are presented in a grid structure according to their time and duration, in which the horizontal axis denotes day, month and year, while the vertical axis indicates the 24 hours of a day. As in many previous works [WPS*09, KSTM10, GS14, BNG12, MLL*13], a straight line could be a natural choice to represent an event, with its length indicating the duration and its width the significance. However, in such a representation, a very significant event with only a short duration would visually appear less significant than a normal event with long duration. This problem cannot be solved by increasing the link thickness due to the notable overlapping caused. Some previous works [BSSB10, DFG12] use a circle to represent events. This can also lead to overlaps if there are many events with long duration. Our solution to this is to use 1) a circle to indicate the significance according to its size (Figure 3) and 2) a line to show the duration. Hence, an event is presented as a glyph which is a combination of a circle and a line.

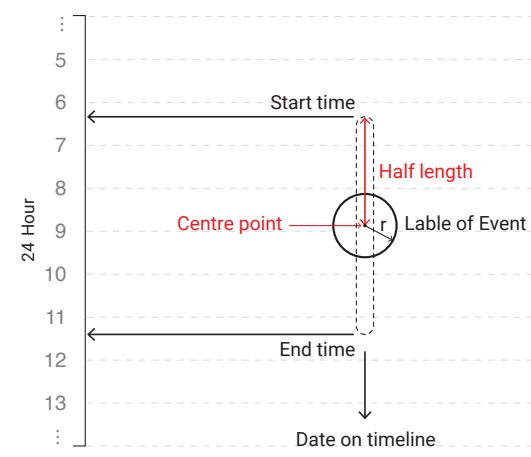

Figure 3: Lines and circles representing the duration and significance ranking of an event. The x-position of the circle shows the date and the y-position shows the hour of the day.

MyMoment: To evoke event familiarity (G2), selected events are presented in a panel called MyMoment (Figure 1-B), showing the contextual information (R7), photos (R8) and statistical information (R9) about the event.

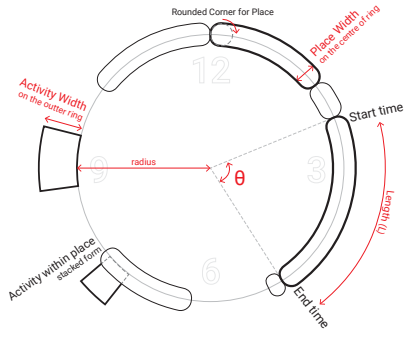

Figure 4: circular layout pictures a selected event in the context of the day when it happened, together with other events in two radial AM/PM clocks

The contextual details include map, time and duration, weather and day overview. Apart from the weather information, the rest of the details can be directly extracted from the data itself. The weather information is obtained by passing the geo-locations and the time to the available historical weather APIs such as World Weather Online (https://developer.worldweatheronline.com/api/historical-weatherapi.aspx). Sequential events that occurred before and after the selected event are also visualised in lines and circles (Figure 1). ClockView is used to show when the event occurred in the context of the entire day (day overview), using two interactive radial clocks for morning and afternoon. This helps MyEvents to use the limited space within the normal size display and effortlessly show the events and movements that happened on the day with alignment to the time. The events are colour coded according to their categories (Figure 4).

Photos from the event are displayed. These include private photos from users themselves or public photos that show the event venue.

In addition, statistical information is also made available, including the number of occurrences and durations of the event, the histogram of the occurring time (i.e. hour of the day), the weekly occurrence and the annual occurrence.

There is also a narration box for users to enter notes about the event in free-text. The notes together with the event information can be included within the created digital memento, which can subsequently support users in the process of reminiscence.

Memento storyline and memento list: This is a modal window that displays all the saved mementos in two different forms, namely either a grid-based or storyline-based style - see (R6). In the gridbased style, we arrange the saved event mementos in a grid. Each memento carries essential information such as photos, narration, time and location but not statistics. In the storyline-based style, these event mementos are placed along an interactive timeline in a compact form that supports zooming in and out (Figure 5). The modal window is only opened upon user selection, so that the display of the saved mementos does not normally overlap with the main user interface.

Event category legend: The event category legend located at the bottom of the EventLine indicates 1) the categories of the events in 


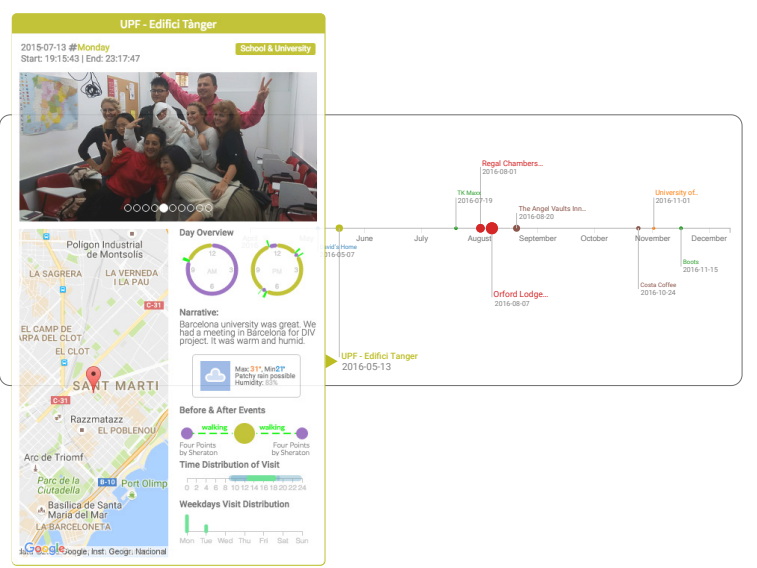

Figure 5: Interactive Memento Storyline shows all the saved mementos along the timeline allowing for interaction to present extra details in a compact form.

different colours, 2) the importance of each category using different heights, and 3) a list of top-ranked places within each category. Thus we assign 9 distinct colours from ColorBrewer [HB03] and also heights to each category to help users differentiate the classification of events and provide information based on the calculated influence factors within the selected time period (see Figure 6).

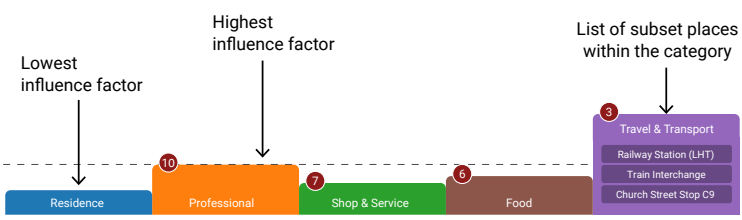

Figure 6: Event category legend

\subsubsection{Interaction}

With the user interface described in Section 3.4.1, MyEvents offers a range of user interactions for data exploration such as overview, query, data upload and saving.

Overview of personal events: By default, MyEvents visualises the personal events in the current year with indications of their significance ranking in the EventLine, allowing the user to gain an overview of all the activities in the year - see R1. Through the multiscale timeline, users are able to select different years.

Event query and exploration: The event query allows users to search for events in the EventLine and the map based on the customisable multi-significance event ranking. This functionality can be evoked by typing a set of keywords for named entities (e.g. event name, category, time, regular or irregular, frequent or infrequent) in the search box (Figure 7) - see R2, or by the control panel - see R3. MyEvents then finds the relevant events and the mRank ranking model computes the significance before they are visualised in the EventLine and maps - see R4. The users can also select top-ranked places in the event category legend.

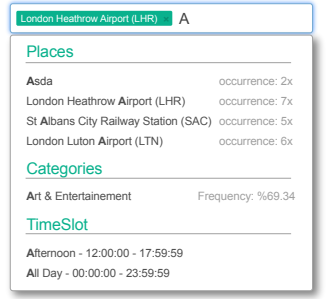

Figure 7: The search box suggests the possible events, categories, or time slot based on the user input (A). The occurrence of the events is also suggested.

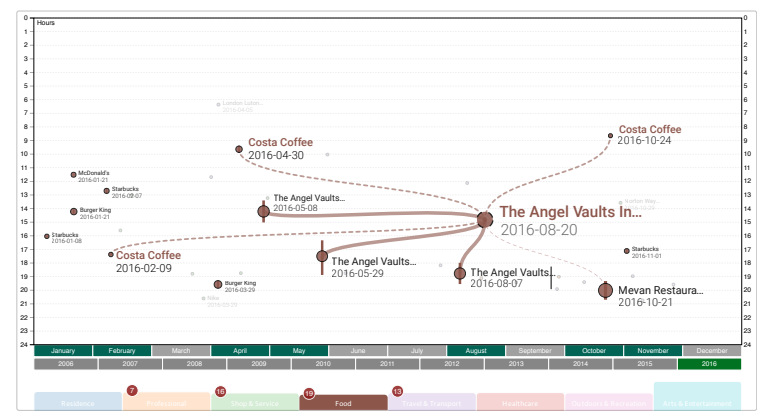

Figure 8: Visualisation of the correlation between events

The correlation between the events in the same category can also be illustrated by hovering the cursor over an event to view the connections; these can be, for example, repeated events, the same place at different locations (e.g. chain shops), and close places within the same category. The width of the connection indicates the geological distance between the places (thicker $=$ closer) while the same places at different locations or the close places are joined with a dashed line - see Figure 8.

Data upload: Data upload is very straightforward. Users need to link their Moves app to MyEvents - see R5. This needs to be done only once, and the data upload becomes automatic when the device is linked to the system.

Mementos viewing, saving, download and retrieval: Users can explore events from the EventLine by clicking on the event circles to see detailed information in MyMoment - see R4. The event mementos can also be saved from MyMoment - see R6. The text box in MyMoment can be used for free-text note writing about the event. The saved mementos can be retrieved by using the memento storyline or the memento list - again see R6.

\section{The Multi-layered significant Event Ranking Model}

This section describes the significant event ranking model (mRank). We first carry out the data preprocessing including data cleaning, data filtering, event extraction, and event validation before passing the data to the mRank model. The preprocessing is performed each time the data changes or loads on the client browser, in order to retain the original data untouched, and to comply with user 


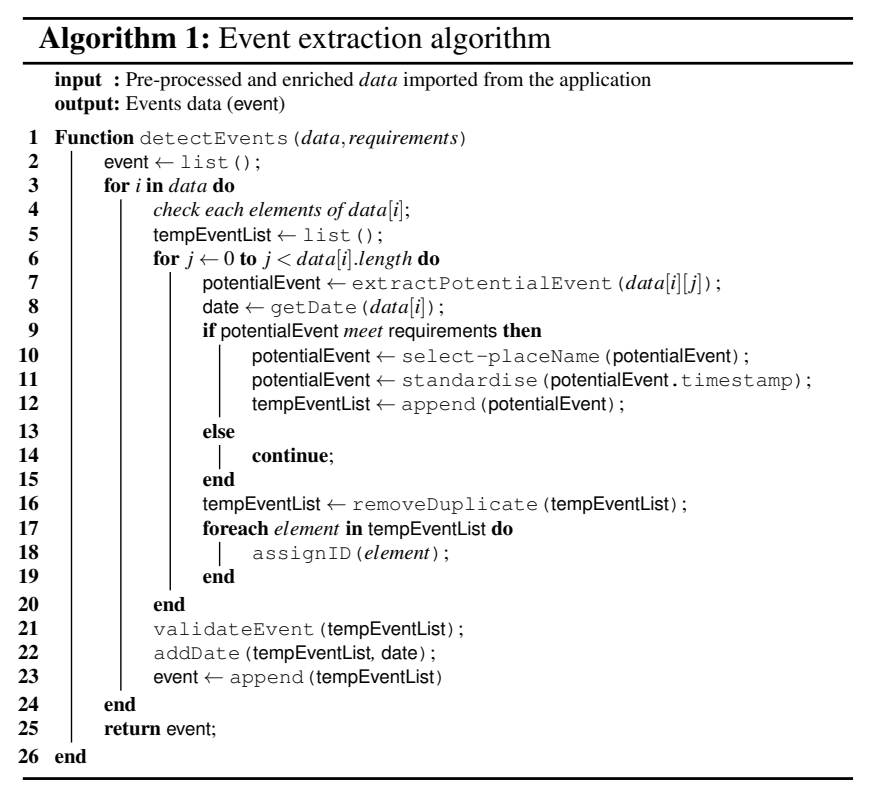

privacy needs. The data cleaning removes errors and noise, while data filtering removes unnecessary entities such as logs, movement and lengthy track points from the raw data. The model initially extracts the candidate events based on the predefined requirements for identifying an event, such as existence of start and end time, coordinate information, and high-level semantics, and then verifies them. A brief outline of the event extraction process is depicted in Algorithm 1.

The model considers three factors in event ranking, including the event category, frequency and regularity. Through the user interface and interaction described in Section 3, users can customise their preferences for these three factors. The importance of these factors is expressed in three weighting coefficients in the model:

- $w_{3}$ : for event category

- $w_{2}$ : for event frequency

- $w_{1}$ : for event regularity

Correspondingly, the ranking score consists of the following three components:

\subsection{Event tf-idf score}

Following the similar concept in text mining, we use the frequency and inverse event frequency scheme to score an event $(e)$ according to its frequency and uniqueness, using the following equation:

$$
\begin{aligned}
\operatorname{tf}-i d f(\mathrm{e})= & {\left[\log \left(1+\frac{f_{e}}{\sum f_{\dot{e}}}\right)\right]^{w_{2}} } \\
& \times \log \left(\frac{|D|}{1+\left|\left\{d_{i} \in D: e \in d_{i}\right\}\right|}\right)
\end{aligned}
$$

where $f_{e}$ is the number of times that the event occurred within a time period, $\sum f_{\hat{e}}$ is the total number of all events within the selected time period, and $w_{2}$ the customised weight of event frequency.
The inverse event frequency is computed as the total number of days in the selected period (e.g. year or month) against the number of the days that the event occurred on in the period. Within the equation, the numerator $|D|$ is the total days or cardinality of $D=d_{1}, d_{2}, \ldots, d_{n}$ where $n$ is the number of days in the selected time period of events. The denominator $\left|\left\{d_{i} \in D: e \in d_{i}\right\}\right|$ is the total number of days within the time period in which the event $e$ occurred.

\subsection{Regularity of event}

The tf-idf weighting represents the global frequency of the events but cannot reflect the magnitude of changes of the data along the time axis. A rare but sudden change, such as moving house, changing work or workplace, is highly valuable in personal event mining but cannot be discovered efficiently by frequency-based tf-idf measures due to their exceptionally low event frequency. To uncover theses sudden changes, the regularity of the events is modelled by using the variance of their occurrence. More specifically, the variance is modelled based on the change of monthly occurrence in the previous 12 months. The variance is given by:

$$
\sigma^{2}(e)=\frac{\sum_{i=1}^{n}\left(f_{i}-\bar{f}\right)^{2}}{n-1}
$$

where $f_{i}$ is the number of occurrences in the event month $i, \bar{f}$ is the average occurrence, and $n$ is the total number of months involved in the calculation for variance. In this work we set $n$ to the last 12 months as this is a reasonable length of time period to estimate the regularity of an event, which is also agreed with by the participants that we have interviewed. The users are more interested in event regularity in recent years rather than in earlier history. Based on the variance, the regularity $\hat{R}(e)$ of the event $\mathrm{e}$ is determined as follows:

$$
\begin{aligned}
\hat{R}(e)= & {\left[\frac{\log \left(\frac{|D|}{1+\left|\left\{d_{i} \in D: e \in d_{i}\right\}\right|}\right)}{\sigma^{2}(e)}\right] } \\
& \times\left(\frac{1}{1+\left|\left\{d_{i} \in D: e \in d_{i}\right\}\right|}\right)
\end{aligned}
$$

Written as:

$$
\hat{R}(e)=\left(\frac{i d f(e)}{\sigma^{2}(e)}\right) \times\left(\frac{1}{d f(e)}\right)
$$

where $i d f_{e}$ is the inverse event frequency, $d f_{e}$ is the number of days that the event occurred in the period, and $\sigma^{2}(e)$ is the calculated variance. This empirical calculation of the regularity favours infrequent but regularly-occurring events by involving $i d f_{e}$ and $d f_{e}$ as the numerators in the equation.

\subsection{Category influence factor}

Since the daily tracking data is semantically enriched with category labels, the category information plays an important role in event 
pattern mining. However, categories should not be treated equally in weighting. The category scoring follows the tf-idf scheme, but in this case the duration of all occurred events in the same category is considered rather than event occurrence, and the score is weighted by $w_{3}$, which is the weighting coefficient for the event category. Using the duration rather than the occurrence of categories has a great impact on the influence factors and can regularise the weight between them. For instance, the influence factor for a workplace category, which normally occurs once a day with a long duration (e.g. 7 - 8 hours) and repeated every day, cannot be the same as the category including short events duration (e.g. 1 hour) with similar or higher occurrence. The influence is given by:

$$
\begin{aligned}
\text { influence }(\text { cat }) & =\log \left(1+\frac{d_{\text {cat }}}{\sum d_{\text {cát }}}\right) \\
& \times \log \left(\frac{|M|}{1+\left|\left\{m_{i} \in M: d_{\text {cat }} \in m_{i}\right\}\right|}\right) \times w_{3}
\end{aligned}
$$

where $d_{\text {cat }}$ is the duration of all events belonging to category (cat) while $\sum d_{\text {cát }}$ is the total duration of the all events in different categories within the time period. The term $|M|$ indicates the number of available months and the denominator $\left|\left\{m_{i} \in M: d_{\text {cat }} \in m_{i}\right\}\right|$ denotes the total number of available months in the time period within which events in category (cat) have occurred. Finally, the multi-significance ranking score of an event is captured by the combination of the above three scores as follows:

$$
\begin{aligned}
S(e \mid d)= & \left(1-w_{1}\right) \times\left[\left(\log \left(1+\frac{f_{e}}{\sum f_{\dot{e}}}\right)\right)^{w_{2}}\right. \\
& \times \log \left(\frac{|D|}{1+\left|\left\{d_{i} \in D: e \in d_{i}\right\}\right|}\right) \\
& \times \log \left(1+\frac{d_{c a t}}{\sum d_{\text {cát }}}\right) \\
& \left.\times \log \left(\frac{|M|}{1+\left|\left\{m_{i} \in M: d_{\text {cat }} \in m_{i}\right\}\right|}\right) \times w_{3}\right] \\
& +w_{1} \times\left[\left(\frac{i d f(e)}{\sigma^{2}(e)}\right) \times\left(\frac{1}{d f(e)}\right)\right]
\end{aligned}
$$

Written:

$$
S(e \mid d)=\left(1-w_{1}\right) \times(\operatorname{tf}-\operatorname{idf}(e) \times \text { influence }(\text { cat }))+w_{1} \times \hat{R}(e)
$$

The implemented algorithm for the significant event ranking is shown in Algorithm 2. The result of this model is a significance score that is pushed into each event as a float value between $(0.0)$ to $(1.0)$.

\section{Evaluation}

In total 16 participants aged between 32 to 50 were recruited with different educational backgrounds for evaluation. All of them had been using the Moves mobile application for a few years with manual annotations to record their daily life activities. The data were

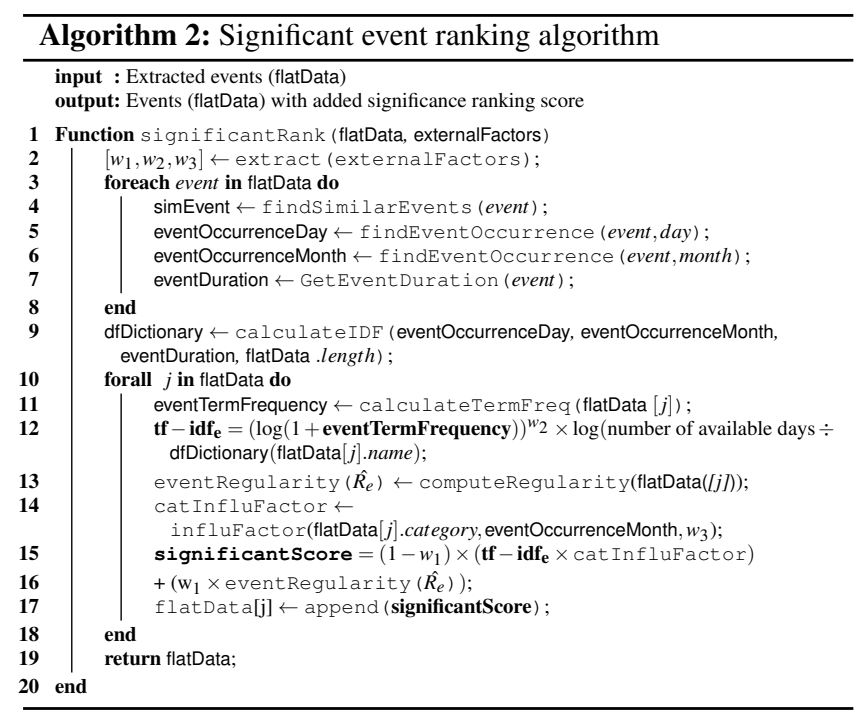

divided according to year and the participants were asked to evaluate different segments of the data.

The evaluation was conducted to examine how effectively MyEvents can support individuals in reminiscence and also to assess its underlying ranking model in terms of accuracy and scalability. The evaluation was carried out by using task completion tests, a usability questionnaire, a user interface satisfaction survey [HSN98], an effectiveness profile questionnaire [Few17], and free text comments. All the participants were provided with an online tutorials, video, and a number of clear examples of how the platform works. Participants were required to upload their data, explore, and interact with the visual interface on their own, and at the end explain their experience. No time limitation to get meaningful insight for reminiscence purposes was imposed. This allowed participants to perform the evaluation with no influence which might have biased the feedback. We conducted an analysis on MyEvents to determine its usability and effectiveness towards supporting reminiscence, and evaluated how our approach helped the participants to complete the tasks. We employed 5-point Likert-type and ranking questionnaires with varying scales ranging from 1 (strongly disagree or low) to 5 (strongly agree or high) to collect user responses.

The significant event ranking model was examined to determine the quality of the results and the performance using real-world datasets. To this end, an initial benchmarking was carried out to create the ground for future methods. The benchmarking of time complexity was conducted by using our own implementation, as well as the JsLitmus.js3 JavaScript library on the Chrome web browser v58, Mac OS 10.12.4, with Intel Core i7 CPU, 32GB memory and Intel 4000 GPU. Subsequently, the quality of the ranking model was evaluated by asking the participants to examine the significance scores of their events based on their own assumption of importance; each event was given a subjective rank score of 1 to 5 . A subjective score of 4 or 5 compared with a calculated score of 0.9 out of 1.0 would indicate that the model provided a sensible score for a fairly significant event. 


\subsection{Tasks}

The participants were asked to complete nine typical tasks related to the design goals of selection subjectivity (G1) and event familiarity (G2). The tasks included including likert-type and open-ended questions, and there was no time limitation. The tasks are listed in Table 2. The purpose of the tasks was to gather the participants' subjective opinions on how MyEvents helped them to complete the tasks based on the design goals and requirements.

The purpose of the task-based evaluation was to instruct the participants to complete certain pre-defined tasks, gain experience, form opinions about the platform, and subsequently score the system performance and provide comments. Tasks T1-T4 were designed to explore personal events by allowing the users to select a time period, together with the use of keywords and other search options. The users could verify the events identified by the platform through visualisation, or search for a remembered event using keywords and check the result against their memory. By doing so, the users could see whether the platform could present events that were perceived as "significant" from their own perspective. Finally they had the opportunity to give feedback about their findings in the questionnaires. Tasks T5-T9 were designed to evaluate some specific functionalities in the platform, including the data upload, saving, event panel, picture and statistics. For the statistics information in $\mathrm{T} 9$, we asked the users to comment on whether the information presented by the platform, such as the average time of visits, average visits during the week, or distribution of the visits over months, were clear and correct from their own judgement and experience.

\subsection{Results}

First, the accuracy and scalability result of our ranking model is provided. We then describe how MyEvents mainly supports personal recall and individual reminiscence, and then show how it addresses the specific goals of selection subjectivity (G1) and event familiarity (G2).

According to the result analysis, the significant ranking model can achieve an average $72.43 \%(\mathrm{SD}=0.07)$ accuracy in determining the event significance. The accuracy is calculated by comparing the participant ranking score (between 1-low to 5-high) to the calculated significant value for a number of their events according to their preferences. Furthermore, we examined the time complexity of the model by measuring the completion time in calculating the significant score with respect to different sizes of dataset (from 2 month to 12 months). The result showed that the time complexity of our ranking algorithm is $O(\log n)$ and by increasing the number of datasets the execution time grows logarithmically - see Figure 9. We also examined the efficiency of the implemented significant ranking algorithm by determining the number of operations per second in 10 different instances. The result of this benchmark showed that our algorithm can run average 42500 operations per second (Figure 10

The user questionnaires showed that our approach is effective in supporting reminiscence by employing data mining models and visualisation approach (Figure 11). $75 \%$ of the participants stated that MyEvents is easy to use and has a user-friendly interface; $69 \%$ of the participants mentioned that the provided information

\begin{tabular}{cl}
\hline Task & Question \\
\hline T1 & $\begin{array}{l}\text { Select different period of time and make observations } \\
\text { to the overviews of all the events. } \\
\text { • Please comment on whether you can gain a good overview of your ac- } \\
\text { tivities within a selected period? }\end{array}$ \\
T2 & Use the search box to discover an event according to \\
location and category. You may also try the keywords \\
of "frequent, rare, regular or irregular". \\
- Please comment on your experience in retrieving a target event using \\
the search box which features incremental search assisted by the clues \\
provided by the system. \\
T3 Use the control panel to discover an event according to \\
the event category in conjunction with the three param- \\
eters ("view more event", "view more regular events", \\
"view more frequent events") which define the impor- \\
tance of an event according to its name, category, date \\
and time together with preference settings. \\
• By doing so, you give more weight to your choice of category (point of \\
interest) and also to the (regular-irregular) or (frequent-rare) events. Please \\
comment on your experience in retrieving target events via the control \\
panel.
\end{tabular}

Table 2: The conclusive evaluation tasks

was sufficient for the purpose of reminiscence whilst $75 \%$ mentioned that the interaction facilitated gaining better understanding. $81 \%$ indicated that this approach enabled them to recall the past by means of interactive visualisation and the provided visual components. Overall feedback showed that $88 \%$ of the participants mentioned that our approach helps the process of reminiscence. In addition, $75 \%$ of participants mentioned that MyEvents can also improve their lifestyle. $69 \%$ of the participants stated that working with MyEvents was interesting and they enjoyed exploring the data and creating event mementos. $94 \%$ of the participants agreed that MyEvents allows the discovery of new knowledge about their own daily lifestyles during the exploration.

The result of assessing the effectiveness in Figure 12 shows that MyEvents is a useful approach to support memory recall from personal daily life data by providing a perceptible and relevant information via an user friendly interface. The evaluation of the effectiveness is inspired by [Few17] in accordance with the informative and emotive terms.

The user interface of MyEvents received a well-grounded feed- 


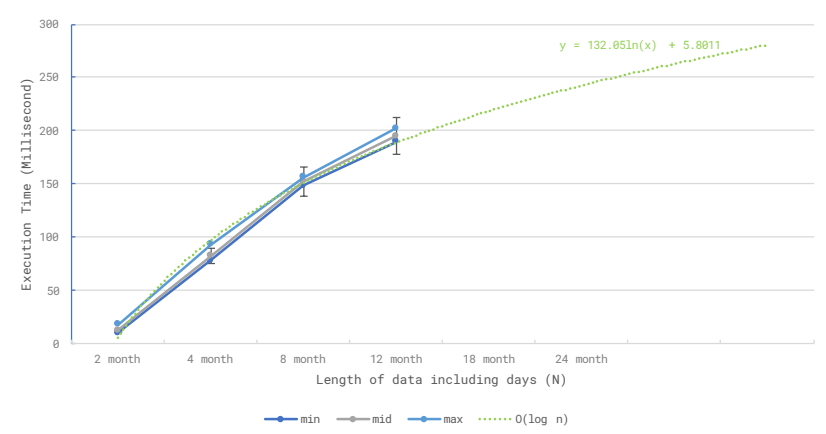

Figure 9: The Multi significant ranking model time complexity

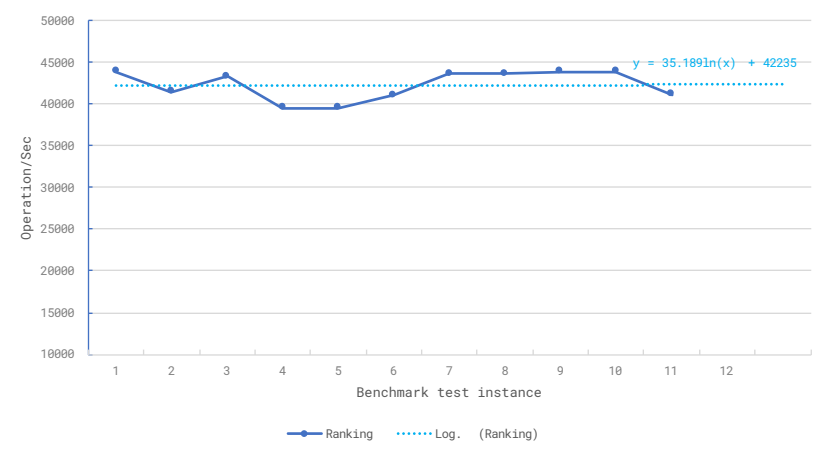

Figure 10: The efficiency of the ranking model based on the operation per second

back (Figure 13). The result shows that the overall reaction to the interface, learning, and system capabilities gained average 4.17/5, $4 / 5$, and $4.02 / 5$ scores respectively. In addition, the screen and terminology achieved a score of $3.83 / 5$, and $3.97 / 5$. The most positive aspects of our approach mentioned by the participants were clarity, simplicity, overall design and interaction between different events whereas the most negative aspects were the lack of known keywords for the search box and confusion between two memento retrieval methods.

\subsubsection{Recall and individual reminiscence}

According to the participants' responses, MyEvents effectively involves the users in the reminiscence process and presents events in a favourable way to evoke event familiarity: "MyEvents gave me a freedom to select an event based on my interest and showed me a gripping overview. I could select an event, view the photos, and write something about it. I was able to show my storyline to my wife and talk about our past!" Another participant stated that our system helped him see a very rare and special event that he had entirely forgotten and then recall the good memory of that: "This system showed me an event that I totally forgot amongst all my day to day events. I then used provided interaction to get more information about it by looking at the photos, map, clock view, etc. It put

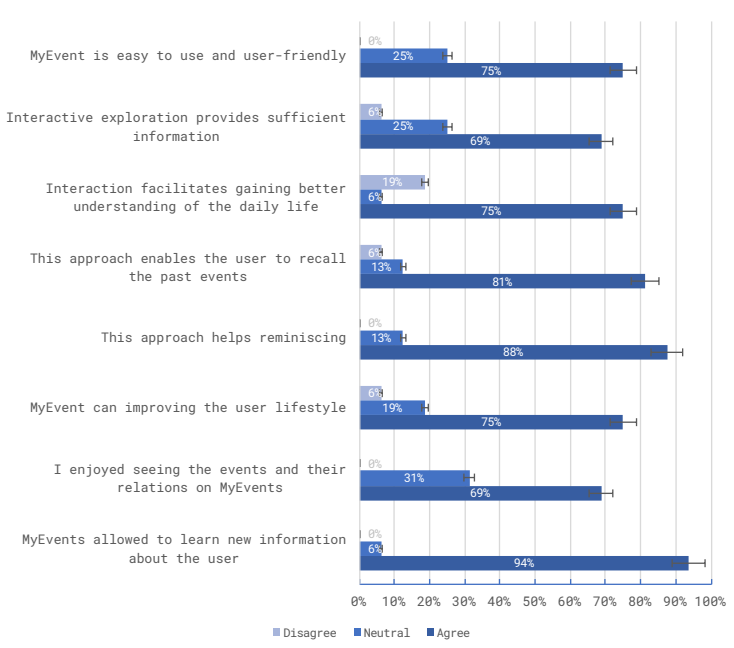

Figure 11: The result of the usability Likert questionnaires

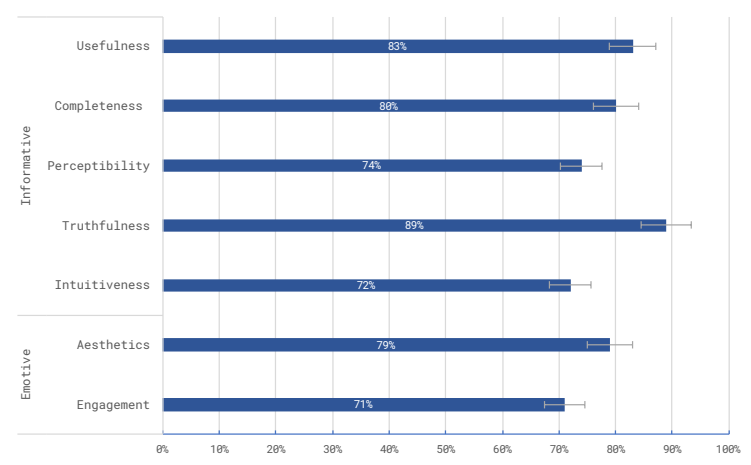

Figure 12: The MyEvents effectiveness profile

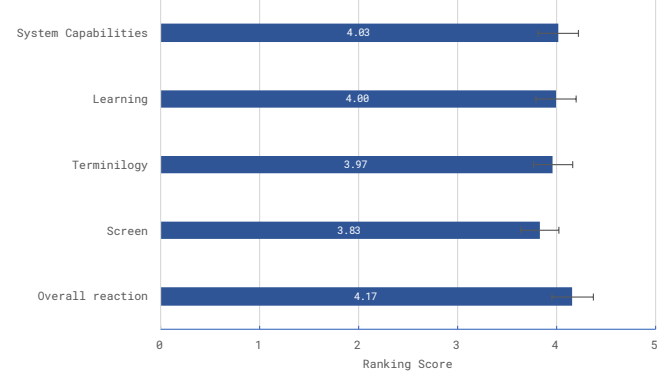

Figure 13: MyEvents user interface satisfaction 
smile on my face when I remembered it." The positive comments show that our system including the ranking model and visualisation highlights significant events in personal history very well.

\subsubsection{Selection subjectivity $($ G1)}

The participants were asked to assess how well the system helped them gain an overview of their own daily data as one of the design requirements. According to the overall feedback, MyEvents provides an appealing overview of daily-life events (R1) with mean $4.35 / 5$. The participants were satisfied with the overview and in some cases they discovered interesting facts about their life: "Overall, MyEvents gives me a good overview at a selected time period and support further interaction to retrieve the events. Having some kind of tooltip to tell you what next within the layout would be great".

To assess how the search box and control panel help retrieve and discover events $(\mathbf{R 2}, \mathbf{R 3})$, we asked the participants to retrieve their own data via the search box and control panel and rate the functionalities accordingly. The feedback shows that the search box is very useful (with mean 4.12/5) whilst the control panel is regarded as a more intuitive way of changing the settings based on the user preferences (mean 4.2/5). However, we learned that there is a need to provide more guidance about available keywords within the search box that can be used for changing the settings as well as filtration: "I had no idea what kind of keyword I can use to search for different things, it was confusing!".

The visualisation of events (R4) achieved an average 4.32/5. The comments were mostly positive, such as "The events are displayed in different size and colours along 24 hours, which was helpful. I also like the category legend, in which I could see the significant places I have been to in each category". Some of the participants wished to see the significance scores inside the event circles. One of our main focuses in the evaluation was to assess the support of the system towards saving, downloading and retrieving of digital mementos (R6); the provided functions were attained average $4.3 / 5$ by the participants. However, offering two different ways of retrieving the mementos caused some confusion despite the layouts being consistent.

\subsubsection{Event familiarity (G2)}

Providing additional information to invoke event familiarity is a vital feature in the system. We have analysed the participants' comments and responses to the Likert-scale questions, to assess how effectively the system can fulfil this. The result reveals that MyEvents satisfies the requirements (R7) by achieving average of 4.4/5: "I like the layout and the way the information like map or time are represented together. This gives me very good assistance for recalling the event in terms of location and date". Although the feedback was positive, we received a few suggestions regarding the use of a bigger geographical map to see more information about the event location.

To support event familiarity, we also allow users to view photos of and statistical information about events. We believe that this information can contribute significantly to support the invocation of memory. According to the evaluation analysis, using photos
(R8) broadly supports the reminiscence process (mean 4.53/5): "I think photos are the most important items that helped me to revoke the old memories". In contrast, using the statistical information is regarded as a useful feature (with mean 4.33/5) for discovering knowledge about events (R9), which can create a more enjoyable experience: "The statistics features are quite interesting although they are not essential! They are mostly educational".

\section{Discussion and Future Work}

MyEvents offers a distinctive way to explore daily personal location and movement data via visual analytics, and supports memory recall by creating event mementos for reminiscence. To identify key events from large personal data, the scalable novel event ranking model, mRank, plays a key role by providing significance ranking based on the three factors of category, frequency and regularity. The significant events in life can be expanded to emotional and social impacts if pertinent data can be obtained. This allows for discovery of events according to user preference, to facilitate user viewing and memento creation. The user interface allows users to interactively set their preferences, either through the control panel or by using the keywords "regular, irregular, frequent or rare" in the search box. This user involvement is key to allowing subjectivity in calculating the event scores. Our research shows that both the search box and control panel have a direct impact on selection subjectivity.

The subjective selectivity is also well-supported by the timelinebased visualisation (i.e. EventLine) and other visual components such as the event category legend. The evaluation shows that the visualisation in MyEvents plays a very positive role in terms of helping the users to select personal events for reminiscence. The visual components in MyEvents allow users to gain a clear overview of personal history, and to easily discover and quickly access a set of events of interest. The interactive visualisation enables users to explore more details of the events, such as obtaining more information, viewing links between events, and visualising event mementos, hence allowing users to find more pertinent and sensible results. Our studies reveal that the participants were interested in using the platform to explicitly explore their own data with the help of the ranking model and the visual components and to create digital mementos in support of reminiscence. They also used MyEvents to attain deeper knowledge about their own daily life.

The presentation of the mementos was effective in terms of supporting memory recall. Collectively, the contextual information, photos and statistics provided valuable information and assisted the users in re-experiencing the past. The memento functions including saving and retrieval were also very useful from the user perspective: firstly, they did not need to search for the same events repeatedly; and secondly, the stored events were made available to the users according to their preferred layout; for example, they could access their recently stored mementos immediately through the grid style, or alternatively, they could view all the stored the mementos in an interactive storyline. 


\subsection{Limitations and future work}

The evaluation and analysis also helped us comprehend a number of limitations of the current approaches in MyEvents and hence, future work can further explore areas such as:

- Automatic place annotation and dynamic event categorisation. MyEvents performs quite well in processing semantically annotated data and generating meaningful visualisation results. The current platform is capable of processing self-annotated data from the Moves app. However, this needs extra effort to manually annotate the places within data for individuals. We concurrently work on the automated place annotation to overcome this limitation by allowing for automatic place annotation and categorisation.

- Time window. MyEvents is designed to show the last 12 months due to the current display viewport limitation. Although allowing a longer period may result in broader exploration and perhaps better knowledge discovery, this may also lead to a cluttered view with numerous overlapping events, which would greatly reduce the effectiveness and user engagement. Similarly, this limitation restricts the category influence factor (category legend) which is following the time window.

- Keyword hints. We learned that users need further assistance in using the correct keywords in the search box. This suggests the need for more hints as well as better guidance within the search box.

- Impact of using multimedia in reminiscence. Using multimedia including video, sound track and music in MyMoment may enhance the presentation of digital mementos and hence facilitate the effectiveness of this approach.

- Additional external factors in the multi-significance event ranking model. Although the current ranking model demonstrated a good result, it might be expanded by considering other external factors in the score calculation, such as emotional and social impact.

- Use in mobile devices. MyEvents is a web-based approach designed for a desktop screen. Future work should address how the layout and visualisation are reformed for the tablet and mobile devices that are used routinely by users.

\section{Conclusion}

This paper presents MyEvents, which adopts a personal visual analytics approach to query, rank, and visualise personal events and support personal reminiscence. We introduced a novel multisignificance mRank event ranking model which takes into account event category, frequency and regularity, allowing for user preferences through either the graphical user interface or using keywords.

MyEvents is designed for normal citizens rather than experts. It fulfils two main goals in reminiscence. The first goal addresses selection subjectivity, which enables users to identify the events of interest by modifying the ranking preferences, making event queries, gaining overviews of personal history and exploring further details about the events through interactive event visualisation along a multiscale timeline. The second goal addresses event familiarity, which evokes memory familiarity by presenting the user's selected event with key contextual information, photos and statistical infor- mation. Users can save and subsequently retrieve and download the mementos.

We evaluated MyEvents by involving a number of participants, all of whom who had multiple years of self-tracking location and movement data. The participants were instructed to complete a set of tasks and a questionnaire and were interviewed. The evaluation result shows that MyEvents effectively supports reminiscence, helps users gain knowledge about themselves from their location and movement data, and presents a sound approach to an interesting research issue in personal visual analytics.

\section{Acknowledgements}

The research is supported by the European Commission within research projects MyHealthAvatar (No 600929), and iManageCancer (No 643529), and by the UK Engineering and Physical Sciences Research Council within research project MyLifeHub (EP/L023830/1).

\section{References}

[AAB*13] Andrienko G., Andrienko N., BAK P., KeIM D., WroBEL S.: Visual Analytics of Movement. Springer, 2013.

[AAFJ16] ANDRIENKo N., ANDRIENKo G., FUCHS G., JANKOWSKI P.: Scalable and privacy-respectful interactive discovery of place semantics from human mobility traces. Information Visualization 15, 2 (2016), 117-153.

[AAH*13] ANDRIENKo G., ANDRIENKo N., HuRTER C., RinZIVILlO S., Wrobel S.: Scalable analysis of movement data for extracting and exploring significant places. IEEE Transactions on Visualization and Computer Graphics 19, 7 (July 2013), 1078-1094.

[AMM*07] Aigner W., Miksch S., Müller W., Schumann H., Tominski C.: Visualizing time-oriented data - A Systematic View. Comput. Graph. 31, 3 (June 2007), 401-409.

[BNG12] Barata G., Nicolau H., Gonçalves D.: AppInsight : What have I been doing? Proceedings of the International Working Conference on Advanced Visual Interfaces (AVI'12) (2012), 465-472.

[BSK05] BRYAnT F. B., Smart C. M., King S. P.: Using the past to enhance the present: Boosting happiness through positive reminiscence. Journal of Happiness Studies 6, 3 (2005), 227-260.

[BSSB10] Baur D., Seiffert F., Sedlmair M., Boring S.: The streams of our lives: Visualizing listening histories in context. IEEE Transactions on Visualization and Computer Graphics 16, 6 (Nov 2010), 1119-1128.

[BWB14] BeEcham R., Wood J., Bowerman A.: Studying commuting behaviours using collaborative visual analytics. Computers, Environment and Urban Systems 47 (2014), 5 - 15. Progress in Movement Analysis - Experiences with Real Data.

[CYW*16] Chen S., YuAn X., Wang Z., Guo C., Liang J., Wang Z., ZHANG X. L., ZHANG J.: Interactive visual discovering of movement patterns from sparsely sampled geo-tagged social media data. IEEE Trans. Vis. Comput. Graph. 22, 1 (2016), 270-279.

[DFG12] DiAs R., FonseCA M., GonçALVES D.: Interactive exploration of music listening histories. Proceedings of the Workshop on Advanced Visual Interfaces AVI (2012), 415-422.

[Few17] FEw S.: Data visualization effectiveness profile, January 2017 https://www.perceptualedge.com/articles/visual_business_intelligence/ data_visualization_effectiveness_profile.pdf; retrieved March 2017.

[FKP*02] Frohlich D., Kuchinsky A., Pering C., Don A., Ariss S.: Requirements for photoware. In Proceedings of the 2002 ACM Conference on Computer Supported Cooperative Work (New York, NY, USA, 2002), CSCW '02, ACM, pp. 166-175. 
[FKSS06] Fails J. A., Karlson A. K., Shahamat L., ShneiderMAN B.: A visual interface for multivariate temporal data: Finding patterns of events across multiple histories. In IEEE VAST (2006), Wong P. C., Keim D. A., (Eds.), IEEE Computer Society, pp. 167-174.

[GS14] Gotz D., Stavropoulos H.: Decisionflow: Visual analytics for high-dimensional temporal event sequence data. IEEE Transactions on Visualization and Computer Graphics 20, 12 (Dec 2014), 1783-1792.

[HB03] Harrower M., Brewer C. A.: ColorBrewer.org: An Online Tool for Selecting Colour Schemes for Maps. The Cartographic Journal 40, 1 (jun 2003), 27-37.

[HK09] Hilliges O., KIRK D. S.: Getting sidetracked: Display design and occasioning photo-talk with the photohelix. In Proceedings of the SIGCHI Conference on Human Factors in Computing Systems (New York, NY, USA, 2009), CHI '09, ACM, pp. 1733-1736.

[HSN98] Harper B., Slaughter L., Norman K.: Questionnaire administration via the WWW : A validation \& reliability study for a user satisfaction questionnaire. In WebNet (1998), vol. 97, pp. 1-4.

[HTA*15] Huang D., Tory M., Adriel Aseniero B., Bartram L., Bateman S., Carpendale S., Tang A., Woodbury R.: Personal Visualization and Personal Visual Analytics. IEEE Transactions on Visualization and Computer Graphics 21, 3 (mar 2015), 420-433.

[HW03] HSIEH H.-F., WANG J.-J.: Effect of reminiscence therapy on depression in older adults: a systematic review. International Journal of Nursing Studies 40, 4 (2003), 335 - 345.

[KSDK11] Koh L. C., Slingsb Y A., Dykes J., Kam T. S.: Developing and applying a user-centered model for the design and implementation of information visualization tools. In 2011 15th International Conference on Information Visualisation (July 2011), pp. 90-95.

[KSTM10] Klimov D., Shahar Y., TAIEB-Maimon M.: Intelligent visualization and exploration of time-oriented data of multiple patients. Artif. Intell. Med. 49, 1 (May 2010), 11-31.

[KTE14] KRUGER R., THOM D., ERTL T.: Visual analysis of movement behavior using web data for context enrichment. In PacificVis (2014), Fujishiro I., Brandes U., Hagen H., Takahashi S., (Eds.), IEEE Computer Society, pp. 193-200.

[KW11] Kalnikaite V., WhitTaker S.: A saunter down memory lane: Digital reflection on personal mementos. International Journal of Human-Computer Studies 69, 5 (2011), 298-310. Designing for Reflection on Personal Experience.

[Lew71] LEWIS C. N.: Reminiscing and self-concept in old age. Journal of Gerontology 26, 2 (1971), 240-243.

[MLL*13] Monroe M., Lan R., Lee H., Plaisant C., ShneiderMAN B.: Temporal event sequence simplification. IEEE Transactions on Visualization and Computer Graphics 19, 12 (Dec 2013), 2227-2236.

[NGN08] Nunes M., Greenberg S., Neustaedter C.: Sharing digital photographs in the home through physical mementos, souvenirs, and keepsakes. In Proceedings of the 7th ACM Conference on Designing Interactive Systems (New York, NY, USA, 2008), DIS '08, ACM, pp. 250260.

[Oy12] OY P.: Moves. https://www.moves-app.com/, 2012 Accessed on March 21, 2014.

[PBW14] Petrelli D., Bowen S., Whittaker S.: Photo mementos: Designing digital media to represent ourselves at home. International Journal of Human-Computer Studies 72, 3 (2014), 320 - 336.

[PMR*96] Plaisant C., Milash B., Rose A., Widoff S., ShneiDERMAN B.: Lifelines: Visualizing personal histories. In Proceedings of the SIGCHI Conference on Human Factors in Computing Systems (New York, NY, USA, 1996), CHI '96, ACM, pp. 221-227.

[PSM07] Pousman Z., Stasko J. T., Mateas M.: Casual information visualization: Depictions of data in everyday life. IEEE Transactions on Visualization and Computer Graphics 13, 6 (nov 2007), 1145-1152.

[PW10] Petrelli D., WhitTaker S.: Family memories in the home: Contrasting physical and digital mementos. Personal Ubiquitous Comput. 14, 2 (Feb. 2010), 153-169.
[PWB08] PETRELLI D., WHITTAKER S., BROCKMEIER J.: Autotopography: What can physical mementos tell us about digital memories? In Proceedings of the SIGCHI Conference on Human Factors in Computing Systems (New York, NY, USA, 2008), CHI '08, ACM, pp. 53-62.

[RCDH03] Ringel M., Cutrell E., Dumais S. T., Horvitz E.: Milestones in Time: The Value of Landmarks in Retrieving Information from Personal Stores. In INTERACT (2003).

[RHF05] Riehmann P., HANFler M., Froehlich B.: Interactive sankey diagrams. In Proc. IEEE Symposium on Infomation Visualization, InfoVis 2005 (2005), IEEE, pp. 233-240.

[RWA*13] Rind A., WANG T. D., Aigner W., Miksch S., WongSuphasawat K., Plaisant C., Shneiderman B.: Interactive information visualization to explore and query electronic health records. Found. Trends Hum.-Comput. Interact. 5, 3 (Feb. 2013), 207-298.

[SC99] SHAHAR Y., CHENG C.: Intelligent visualization and exploration of time-oriented clinical data. In Proceedings of the 32nd Hawaii International Conference on System Science, volume Track4 (Jan 1999).

[SGBBT06] Shahar Y., Goren-Bar D., Boaz D., TAhan G.: Distributed, intelligent, interactive visualization and exploration of timeoriented clinical data and their abstractions. Artif. Intell. Med. 38, 2 (Oct. 2006), 115-135.

[Shn96] Shneiderman B.: The eyes have it: A task by data type taxonomy for information visualizations. In Visual Languages, 1996. Proceedings., IEEE Symposium on (Sep 1996), pp. 336-343.

[SMM12] Sedlmair M., Meyer M., Munzner T: Design study methodology: Reflections from the trenches and the stacks. IEEE Transactions on Visualization and Computer Graphics 18, 12 (Dec 2012), 2431-2440.

[TBHC16] Thudt A., Baur D., Huron S., CARpendale S.: Visua mementos: Reflecting memories with personal data. IEEE Transactions on Visualization and Computer Graphics 22, 1 (Jan 2016), 369-378.

[vdH14] VAN DEN Hoven E.: A future-proof past: Designing for remembering experiences. Memory Studies 7, 3 (2014), 370-384.

[VEC09] Vrotsou K., Ellegard K., Cooper M.: Exploring time diaries using semi-automated activity pattern extraction. electronic International Journal of Time Use Research 6, 1 (2009), 1-25.

[VGD06] ViÉGAS F., Golder S., DonATH J.: Visualizing email content: portraying relationships from conversational histories. Proceedings of the SIGCHI conference on Human Factors in computing systems (2006), 979-988.

[VJC09] VRotsou K., Johansson J., Cooper M.: Activitree: Interactive visual exploration of sequences in event-based data using graph similarity. IEEE Transactions on Visualization and Computer Graphics 15, 6 (2009), 945-952.

[vLBR*16] VON LANDESBERGER T,, BRODKORB F., ROSKOSCH P. Andrienko N., ANdrienko G., Kerren A.: Mobilitygraphs: Visual analysis of mass mobility dynamics via spatio-temporal graphs and clustering. IEEE Transactions on Visualization and Computer Graphics 22, 1 (Jan 2016), 11-20.

[WBC10] Whittaker S., Bergman O., Clough P.: Easy on that trigger dad: a study of long term family photo retrieval. Personal and Ubiquitous Computing 14, 1 (2010), 31-43.

[WBH15] West V. L., Borland D., Hammond W. E.: Innovative information visualization of electronic health record data: a systematic review. Journal of the American Medical Informatics Association 22, 2 (Mar. 2015), 330-339.

[WG12] WongsuphasaWAT K., Gotz D.: Exploring flow, factors, and outcomes of temporal event sequences with the outflow visualization. IEEE Transactions on Visualization and Computer Graphics 18, 12 (Dec. 2012), 2659-2668.

[WGGP*11] WONGSUPhasawat K., GuerRa Gómez J. A. Plaisant C., Wang T. D., TAieb-Maimon M., Shneiderman B.: Lifeflow: Visualizing an overview of event sequences. In Proceedings 
of the SIGCHI Conference on Human Factors in Computing Systems (New York, NY, USA, 2011), CHI '11, ACM, pp. 1747-1756.

[WKP*12] Whittaker S., Kalnikaite V., Petrelli D., Sellen A., Villar N., Bergman O., Clough P., Brockmeier J.: Sociotechnical lifelogging: Deriving design principles for a future proof digital past. Human-Computer Interaction 27, 1-2 (2012), 37-62.

[WM99] Webster J. D., McCall M. E.: Reminiscence functions across adulthood: A replication and extension. Journal of Adult Development 6, 1 (1999), 73-85.

[WPS*09] Wang T. D., Plaisant C., Shneiderman B., Spring N., Roseman D., Marchand G., Mukherjee V., SMith M.: Temporal summaries: Supporting temporal categorical searching, aggregation and comparison. IEEE Transactions on Visualization and Computer Graphics 15, 6 (Nov. 2009), 1049-1056.

[YWL*15] YU L., WU W., LI X., LI G., NG W. S., NG S.-K., HUANG Z., ARUnan A., WatT H. M.: iviztrans: Interactive visual learning for home and work place detection from massive public transportation data. In VAST (2015), Chen M., Andrienko G. L., (Eds.), IEEE Computer Society, pp. $49-56$. 